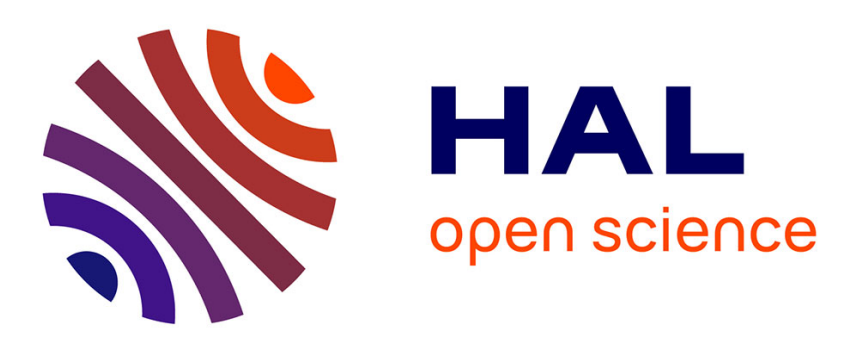

\title{
An Energy-Efficient Addressing Mechanism for Wake-up Radio Systems
}

Jean-Mickaël Lebreton, Nour Murad, Somasekhar Kandukuri, Richard Lorion

\section{To cite this version:}

Jean-Mickä̈l Lebreton, Nour Murad, Somasekhar Kandukuri, Richard Lorion. An Energy-Efficient Addressing Mechanism for Wake-up Radio Systems. IEEE Wireless Telecommunications Symposium (WTS 2016), IEEE Communications Society, Apr 2016, London, United Kingdom. hal-01345001

\section{HAL Id: hal-01345001 https://hal.science/hal-01345001}

Submitted on 10 Nov 2017

HAL is a multi-disciplinary open access archive for the deposit and dissemination of scientific research documents, whether they are published or not. The documents may come from teaching and research institutions in France or abroad, or from public or private research centers.
L'archive ouverte pluridisciplinaire HAL, est destinée au dépôt et à la diffusion de documents scientifiques de niveau recherche, publiés ou non, émanant des établissements d'enseignement et de recherche français ou étrangers, des laboratoires publics ou privés. 


\title{
An Energy-Efficient Addressing Mechanism for Wake-up Radio Systems
}

\author{
Jean Lebreton, Nour Murad, Somasekhar Kandukuri, and Richard Lorion \\ University of La Reunion, LE2P \\ 40 Avenue de Soweto, 97410 Saint-Pierre \\ Email: jean.lebreton@univ-reunion.fr
}

\begin{abstract}
Wake-up Radio receivers (WuRx) must be energyefficient for their integration into Wireless Sensor Networks (WSNs). The most energy-hungry component is the address decoding hardware and it is generally powered by the node battery. In this paper, an addressing mechanism is proposed and the decoding process is explained in detail, on the contrary of previous works. This paper also shows the energy reduction of the address decoding stage while performed by a microcontroller, in comparison with one of the best correlator approach. The energy consumption and latency are also well investigated with real measurements, which is often omitted in previous works. Our approach is also flexible since a trade-off between energy consumption and wake-up latency is proposed. While decoding the address, the most energy-efficient mode consumes only $\mathbf{2 3 . 8}$ $\mu \mathrm{A}$ with a wake-up latency of $8.88 \mathrm{~ms}$.
\end{abstract}

\section{INTRODUCTION}

Wireless Sensor Networks (WSNs) are considered as one of the most promising technologies. Energy efficiency is very crucial in WSN since nodes are usually battery operated devices. In most application scenarios, the major source of energy depletion is the radio module and many research works tends to reduce its energy consumption [1].

Wake-up Radio (WuR) systems have been proposed during the last decade to improve the energy efficiency of Wireless Sensor Networks (WSNs). WuR systems tend to replace duty-cycled Medium Access Control (MAC) protocols, since they reduce significantly the problem of idle listening and overhearing, which are the major sources of energy waste in WSNs [2]. In order to eliminate idle listening, the Wakeup Radio receiver (WuRx) enables the nodes to wake up on demand by a particular radio message, namely a Wake-up Call (WuC). Upon reception of a $\mathrm{WuC}$, the node turns on its main MicroController Unit (MCU) and its main radio card for sending measured data.

Addressing mechanisms can also be integrated into WuRx for selecting a node to wake up, thus reducing overhearing. The addressing functionality requires additional power consumption for the WuRx when it is decoding the address. This energy consumption is often not mentioned in earlier works, but it is crucial for realistic WSN simulations involving WuRx scenarios such in [3]

In this paper, we present a semi-passive WuRx based on the combination of a compact rectenna array [4] and a MCU. The MCU is essentially used to decode the address included in the $\mathrm{WuC}$. Moreover, additional information can be added in the $\mathrm{WuC}$ for developing more sophisticated wake-up schemes. We also measured and analysed the MCU energy consumption while processing the $\mathrm{WuC}$ signal under different oscillator configurations.

Main contributions of this paper are the following:

- we present in detail the addressing mechanism and in particular how the address is decoded by the WuRx;

- we experimentally measure the energy consumption and wake-up latency of the proposed WuRx regarding considering configurations of the MCU;

- we make a comparative analysis of our addressing mechanism with up-to-date approaches.

The rest of this article is organized as follows: in section II we describes related work. Section III presents the hardware design related to the WuR system and also the software developed for the addressing mechanism. The method for measuring the energy consumption and wake-up latency is then described in section IV. Results and discussion are given in section $\mathrm{V}$ for different configurations of the MCU. Section VI presents a comparative analysis regarding the WuRx power consumption. Finally, conclusion and perspectives are given in section VII.

\section{RELATED WORKS}

$\mathrm{Gu}$ and Stankovic proposed the first wake-up radio design with a basic radio-triggered circuit in [5]. Several wake-up receivers have been proposed in the literature to reduce the current consumption of the wake-up radio circuit, and they are classified in different types such as RFID, heterodyne, MCU or correlator.

Many papers proposing a WuRx supporting an addressing mechanism generally focus on the hardware and they give little or no information on the address decoding stage, especially how it is performed and how much it consumes [6]. In this paper, the addressing mechanism is explained in details with the communication protocol used to decode the address. Also, the energy consumption for decoding the address is provided here.

While some previous works used the same modulation technique as our work (OOK modulation) and the same hardware for address decoding (MCU), the decoding technique is still not detailed. For instance, a MCU is used to sample data in [7]-[9], which means they would read the digital port and reconstruct the signal after that. To the best of our 


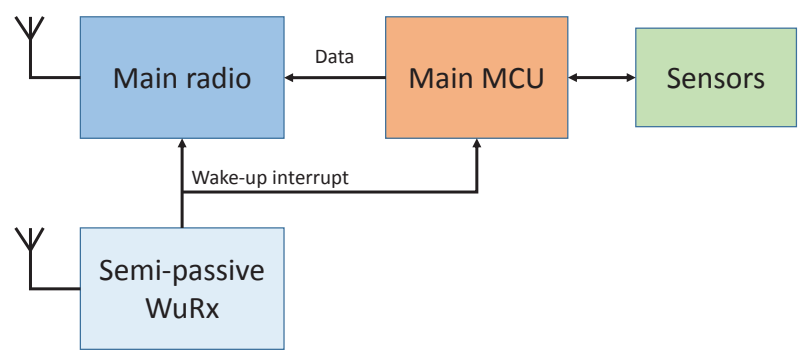

Fig. 1. Block diagram of the node with an additional WuRx circuit.

knowledge, there is no paper proposing a standard or implementing existing communication protocols such as UART into the WuRx for the addressing decoding. Our decoding method is more efficient and also easier to reproduce for further implementations.

For decoding the address, WuRx available in the literature either have a dedicated component or the task is processed by the MCU. While using a dedicated component such as correlator in [10], the power consumption is well investigated. However, the MCU power consumption is lightly considered while being used for the address decoding, such in [7], [9]. In general, a high clock frequency (typically from 1 to 16 $\mathrm{MHz}$ ) is used and only the current consumption in sleeping mode is given, which is not sufficient. This paper shows the combined effect of the clock frequency and the WuC bit rate on the MCU power consumption. Only the work from [11] presented its MCU energy consumption but the approach showed a relatively high power consumption.

\section{HARdwARE Design AND Software Development}

The hardware and software tools implemented for this work are described in this section. The WuRx design is presented first and the related addressing mechanism is then discussed. Finally, our Wake-up Transmitter (WuTx) in charge of transmitting the $\mathrm{WuC}$ is presented.

\section{A. Semi-passive WuRx design}

The considered node structure is first described here with the block diagram depicted in figure 1. A semi-passive WuRx that can harvest energy from received signals is connected to both main radio and main MCU. These two components can be separated devices or they can be integrated into one device as a wireless mote such as TelosB, Xbee or Waspmote. Both of them can be waken up by the WuRx through an interrupt signal.

Our semi-passive WuRx is composed of three main blocks as illustrated in figure 2. The RF energy harvesting circuit converts the RF signal into a DC signal. In this work, we use a compact rectenna array presented in [4] which can harvest energy from received signals in the $2.4 \mathrm{GHz}$ band. A comparator is added to the output of the RF energy harvesting circuit, which then produces a digital "high" if the output DC voltage is greater than a predefined threshold. In our

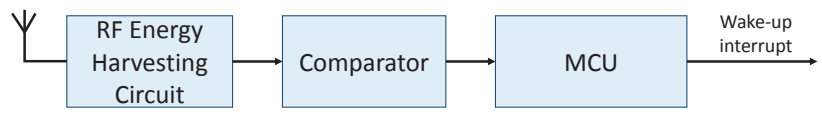

Fig. 2. Block diagram of the semi-passive WuRx.

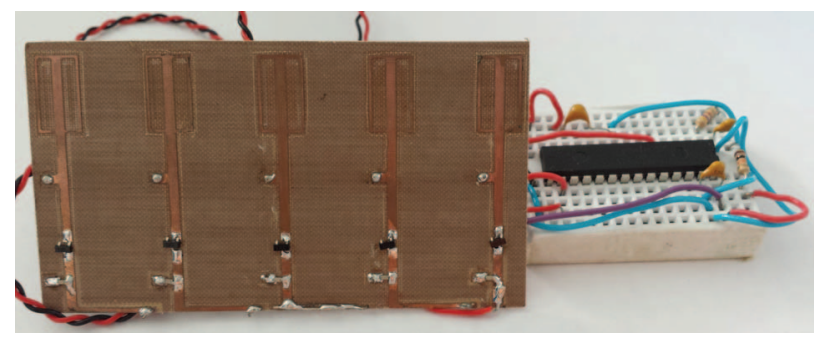

Fig. 3. Picture of the WuRx prototype.

case, a nano-power comparator from Texas Instruments [12] is considered. The comparator output voltage is then connected to a low-power MCU, a PIC24F16KA102 from Microchip [13] in our case. The MCU purpose is essentially to process the address decoding function, and also to generate the wake-up interrupt accordingly for waking up the main MCU and main radio of the node. The WuRx prototype is depicted in figure 3, it was developed and tested in a breadboard. The realization of a PCB (Printed Circuit Board) is currently under consideration.

\section{B. Energy Efficient Addressing Mechanism}

In this paper, addressing mechanism refers to the modulation technique and the communication protocol used for transmitting and decoding the address in the WuC. As the WuRx architecture is relatively simple compared to traditional wireless receiver, there is less possibilities for the addressing mechanism. The simple architecture is also preferred for its low energy consumption since a more complex circuit often require more power consumption.

The most appropriate modulation technique for $\mathrm{WuRx}$ is certainly the On-Off Keying (OOK) and it is also the most employed technique in WuRx. OOK is actually the simplest form of Amplitude-Shift Keying (ASK) modulation where digital data are represented by the presence or absence of a carrier wave. In general, the absence of a carrier wave corresponds to a binary 0 and the presence of a carrier wave corresponds to a binary 1 . The WuRx circuit is compatible with such modulation since the RF energy harvesting circuit converts RF signals into a DC voltage, meaning that the absence of a carrier wave corresponds to a zero voltage while the presence of a carrier wave corresponds to non-zero voltage. The comparator is then employed to reconstruct a digital signal for the MCU.

Some serial communication protocols are already implemented in the MCU for communicating with other peripheral devices such as SPI (Serial Peripheral Interface), UART (Universal Asynchronous Receiver Transmitter) or $\mathrm{I}^{2} \mathrm{C}$ (InterIntegrated Circuit). A UART communication is chosen here 


\begin{tabular}{|c|c|c|c|}
\hline Preamble & Start bit & Destination address & Stop bit \\
$\underbrace{111111111111111}_{N_{\text {pre }} \text { bits }} 0$ & 0 & 10101100 & 0
\end{tabular}

Fig. 4. Frame structure of the $\mathrm{WuC}$ with an example in binary representation, destination address is the symbol ' 5 ' in ASCII table.

due to its asynchronous aspect and the fact that one signal is sufficient for communicating. We then configured the UART communication using one start bit, one stop bit, 8 bits of data and no parity. As the destination address field is composed of 8 bits, the network size limit is 256 nodes. This number of nodes is sufficient for medium size WSNs in which WuR systems are involved. The bit rate, also known as baud rate, is variable since we consider different bit rates for the energy consumption analysis.

Finally, the WuC message is composed of a preamble, a start bit, a destination address field and a stop bit, as illustrated in figure 4. The MCU wakes up from deep sleep mode after detection of the preamble, which must be long enough to cover the MCU oscillator start-up time.

\section{WuTx design}

The challenge is then to develop a Wake-up Transmitter (WuTx) capable of transmitting the WuC according to an OOK modulation and compliant with the UART protocol. Our WuTx is actually a USRP-2922 (Universal Software Radio Peripheral) from National Instruments, which enables the development of a specific physical layer. We implemented a Virtual Instrument (VI) under LabVIEW for prototyping the physical layer communication. The VI main input parameters are the preamble size, the 8-bit destination address and the transmission gain. The VI configures the USRP to transmit the defined message using an OOK modulation with specified parameters such as the carrier frequency fixed at $2.45 \mathrm{GHz}$ and the bit rate.

The VI under LabVIEW and the USRP form together a Software Defined Radio (SDR). The implementation described here refers to the physical layer of the WuTx, but a MAC layer has also been implemented in the same VI for designing the base station related to our network, since the 8-bit address can be dynamically varied in the program. Multiple nodes can then be interrogated by the WuTx and accordingly to the MAC layer specifications, which is not the topic of this paper.

\section{Measurement method}

The measurement system consisted of a variable resistor connected in series with a DC power supply and the MCU. An oscilloscope along with three probes were used to capture the signals related to our wake-up procedure. The first probe (yellow wire) was connected to the variable resistor terminals for measuring the current consumption. The second probe (green wire) was connected to the output voltage of the rectenna for capturing the $\mathrm{WuC}$ reception. The third probe

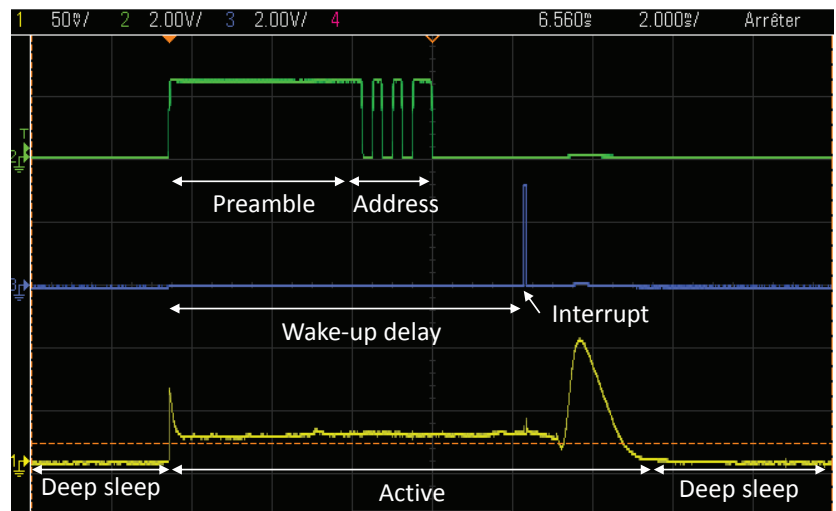

Fig. 6. Signals measured by the oscilloscope upon reception of a WuC. The horizontal sweep is $2 \mathrm{~ms} / \mathrm{div}$, the vertical deflection is $50 \mathrm{mV} / \mathrm{div}$ for channel 1 and $2 \mathrm{~V} / \mathrm{div}$ for channel 2-3.

(blue wire) was connected to the digital port RA0, which is programmed to be driven high upon a WuC validation. The circuit schematic is depicted in figure 5.

The measurement method was carried out for five MCU configurations. A special attention was paid to the system clock source since three internal oscillators are available in the PIC24F. The oscillators frequency can be selected by a programmable clock divider with a nominal $8 \mathrm{MHz}$ output for the Fast internal RC (FRC) oscillator, a nominal $500 \mathrm{kHz}$ for the Lower Power FRC (LPFRC) oscillator and a fixed $32 \mathrm{kHz}$ for the Low-Power internal RC (LPRC) oscillator.

The measurement method is presented for the configuration using the LPRC oscillator at $32 \mathrm{kHz}$. The signals were captured by the oscilloscope upon reception of a $\mathrm{WuC}$, as shown in figure 6. The MCU exits from deep sleep mode immediately after detecting the preamble. A long preamble of 20 bits is chosen here to match with the oscillator startup time and the number of instructions within the program initialization. After the address decoding and validation, the digital pin RA0 is driven high to trigger the node wake-up. The MCU enters deep sleep mode after this interrupt, with a noticeable current peak depicted in figure 6 .

The wake-up delay is defined as the duration between the first bit of preamble received and the interrupt sent by the MCU. Considering this definition, the wake-up delay is directly measured through the oscilloscope.

The MCU energy consumption is calculated by integrating its power consumption over time, which is expressed by equation (1).

$$
E=\int_{0}^{T_{a c t}}\left(U_{G e n}-U(t)\right) \times \frac{U(t)}{R_{v}} d t
$$

where $T_{a c t}$ is the MCU active period, $R_{v}$ is the variable resistance, $U(t)$ is the voltage across the resistor and $U_{G e n}$ is the voltage of the DC power supply.

The resistance $R_{v}$ is selected accordingly to get a voltage $U(t)$ negligible compared to $U_{G e n}$. The equation (1) is then 


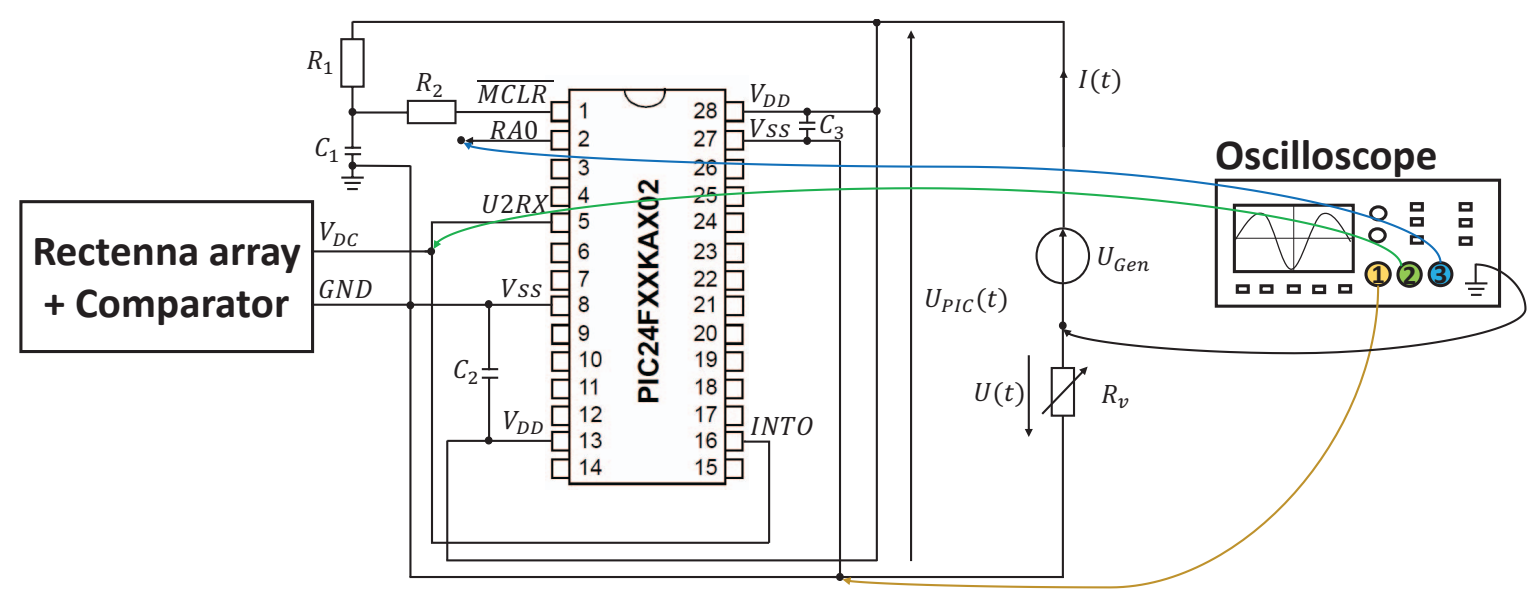

Fig. 5. Energy measurement system applied to the wake-up radio

simplified into equation (2) assuming that $U(t)<<U_{G e n}$.

$$
E=\frac{U_{G e n}}{R_{v}} \int_{0}^{T_{a c t}} U(t) d t
$$

The oscilloscope computed the numerical integration of $U(t)$ over the active period $T_{a c t}$ with a high resolution of 2.5 million samples per second.

\section{RESUlts AND Discussion}

For each configuration, we empirically determined the minimum preamble size. For smaller size of preamble, the MCU does not process the address decoding correctly. The minimum preamble size is then selected in each configuration for energyefficiency purpose. The bit-rate is often very limited in WuRx systems. Our rectifying circuit would theoretically be able to reach a bit-rate of $10.4 \mathrm{kbps}$, but a practical limit of $8 \mathrm{kbps}$ was noticed. We varied the bit-rate between 4 and $8 \mathrm{kbps}$, which is the same order as other WuRx systems. The experimental results are given in table I.

The first two scenarios show the clear impact of the bit rate on both energy consumption and wake-up delay while using the FRC oscillator. A higher bit rate gives a lower wake-up delay and lower energy consumption. In this case, the energy consumption is reduced because of the lower wake-up delay since the current consumption is almost same for both scenarios.

Two other scenarios were considered while using the LPFRC oscillator, in which they differ from the frequency selection. This case is particularly interesting because it presents a trade-off between energy consumption and wake-up delay. The MCU consumes $1.79 \mu \mathrm{J}$ with the LPFRC oscillator set to $500 \mathrm{kHz}$ while it consumes $1.71 \mu \mathrm{J}$ with the LPFRC oscillator set to $62.5 \mathrm{kHz}$. However, the wake-up delay is only equal to 1.94 ms with the $500 \mathrm{kHz}$ oscillator while it is equal to 3.42 ms with the $62.5 \mathrm{kHz}$ oscillator. Even if our main motivation is energy-efficiency, we notice an energy reduction of $5 \%$ with a wake-up delay increased by $76 \%$. This trade-off is not advantageous for applications involving in both energy and latency constraints.

The LPRC oscillator is selected in the last scenario. The maximum bit rate of the serial communication is equal to one eighth of the oscillator frequency. The optimal bit rate is then considered for this configuration. The energy consumption is lower than the other configurations but the wake-up delay is longer, as it consumes only $1.01 \mu \mathrm{J}$ with a wake-up delay of $8.88 \mathrm{~ms}$.

For our wake-up system, we obviously pick the $32 \mathrm{kHz}$ LPRC oscillator since it is the most energy-efficient. If a trade-off between energy and latency is required, the $500 \mathrm{kHz}$ LPFRC is certainly the best choice. Compared to the LPRC oscillator, it reduces the wake-up delay by a factor of 4.5 while only increasing the energy consumption by a factor of 0.8 .

\section{COMPARATIVE ANALYSIS}

Hardware correlators are often presented as one of the most energy-efficient component for addressing purpose and several WuRx in the literature are based on correlator. The WuRx in [10] was presented as one of best WuRx in the literature. Its power consumption was well studied, allowing an accurate comparison with real measures. A MCU running at $8 \mathrm{MHz}$ was used in [7] but the energy consumption for address decoding was not provided, our measures are then exploited for comparison. Our MCU approach using the 500 $\mathrm{kHz}$ LPFRC oscillator or the $32 \mathrm{kHZ}$ LPRC oscillator are then compared to their approach.

The comparative analysis is based on the average current consumption of the MCU or correlator. The average current consumption $\bar{I}$ is highly dependent on the frequency of WuC reception, which corresponds to the network traffic. Let us define the $\mathrm{WuC}$ inter arrival time $T_{w}$ as the duration between two consecutive WuC receptions. The average current consumptions are calculated through equation 3 with the 
TABLE I

WAKE-UP DELAY AND ENERGY CONSUMPTION FOR ADDRESS DECODING USING DIFFERENT CONFIGURATIONS OF THE MCU.

\begin{tabular}{llllll}
\hline Oscillator & FRC & \multicolumn{3}{l}{ LPFRC } & LPRC \\
\hline Frequency & $8 \mathrm{MHz}$ & & $500 \mathrm{kHz}$ & $62.5 \mathrm{kHz}$ & $32 \mathrm{kHz}$ \\
Preamble size (bits) & 2 & 2 & 5 & 10 & 20 \\
Bit rate (kbps) & 4 & 8 & 8 & 8 & 4 \\
\hline Wake-up delay (ms) & 2.94 & 1.59 & 1.94 & 3.42 & 8.88 \\
\hline Current consumption $(\mu \mathrm{A})$ & 3196 & 2970 & 181 & 95.5 & 23.8 \\
Energy consumption $(\mu \mathrm{J})$ & 32.7 & 16.6 & 1.79 & 1.71 & 1.01 \\
\hline
\end{tabular}

parameter values given in table II.

$$
\bar{I}=\frac{T_{w}-T_{a c t}}{T_{w}} I_{i d l e}+\frac{T_{a c t}}{T_{w}} I_{a c t}
$$

where $I_{i d l e}$ is the current consumption in idle mode and $I_{\text {act }}$ is the current consumption in active mode for decoding the address.

TABLE II

CURRENT CONSUMPTION PARAMETERS CONSIDERING DIFFERENT HARDWARE APPROACHES.

\begin{tabular}{llll}
\hline Addressing hardware & $I_{\text {idle }}(\mu \mathrm{A})$ & $I_{\text {act }}(\mu \mathrm{A})$ & $T_{\text {act }}(\mathrm{ms})$ \\
\hline Correlator [10] & 2.6 & 8.8 & 10.2 \\
MCU - FRC [7] & 0.2 & 2970 & 1.7 \\
MCU - LPFRC & 0.2 & 181 & 3.3 \\
MCU - LPRC & 0.2 & 23.8 & 12.87 \\
\hline
\end{tabular}

The evolution of the average current consumption $\bar{I}$ is depicted in figure 7 for the four considered approaches. Figure 7 shows that the MCU approach can perform better than the correlator approach from an energy perspective. In fact, the correlator approach consumes less current when the WuC inter arrival time is low, which corresponds to a WuRx requested very frequently. As the correlator consumes less current in its active mode compared to the $\mathrm{MCU}$, the average current consumption in then lower for this scenario. However, WuRx are preferably used in low duty-cycle WSN where nodes are most of the time in deep sleep mode. For this reason, the MCU approach is more energy-efficient than the correlator approach due to its very low idle current. The average current consumption is also reduced by using the MCU low-power modes for address decoding, compared to the $8 \mathrm{Mhz}$ mode in [7].

\section{CONCLUSIONS AND PERSPECTIVES}

An energy-efficient addressing mechanism is proposed for WuRx in WSNs. Even though this addressing mechanism was applied to our WuRx, it can be integrated in any WuRx of the literature for reducing their energy consumption. The energyefficiency is obtained by optimizing the MCU configuration for the specific task of decoding the address included in the WuC. The energy consumption can also be trade off for latency with this MCU approach, since different power modes are available.

The presented addressing mechanism can also be used to send additional data directly from the WuTx to the WuRx,

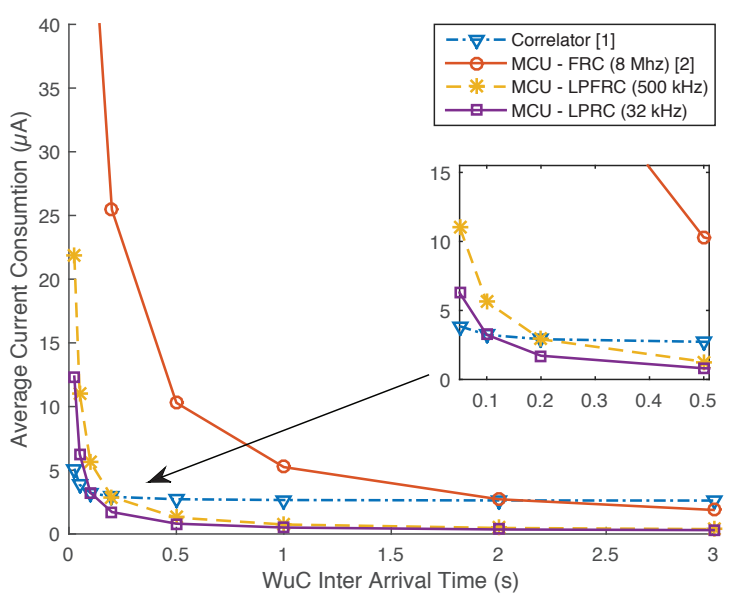

Fig. 7. Average current consumption vs. WuC inter arrival time.

which might be useful for a more complex WuR protocol. As the first block of our WuRx design is an energy harvesting circuit, this work can be a supportive tool for the growing field of Simultaneous Wireless Information and Power Transfert (SWIPT).

\section{REFERENCES}

[1] T. Rault, A. Bouabdallah, and Y. Challal, "Energy efficiency in wireless sensor networks: A top-down survey," Computer Networks, vol. 67, pp. 104-122, July 2014.

[2] J. Oller, I. Demirkol, J. Casademont, J. Paradells, G. U. Gamm, and L. Reindl, "Has Time Come to Switch From Duty-Cycled MAC Protocols to Wake-Up Radio for Wireless Sensor Networks?," IEEE/ACM Transactions on Networking, pp. 1-1, 2015.

[3] J. Lebreton and N. Murad, "Implementation of a Wake-up Radio Cross-Layer Protocol in OMNeT++/MiXiM," in Proceedings of the 2nd OMNeT++ Community Summit, Sept. 2015.

[4] J. Riviere, A. Douyere, and J. D. Lan Sun Luk, "Impact of the coupling effect and the configuration on a compact rectenna array," in IOP Conference Series: Material Science and Engineering, vol. 67, 2014.

[5] L. Gu and J. A. Stankovic, "Radio-Triggered Wake-Up Capability for Sensor Networks.," in IEEE Real-Time and Embedded Technology and Applications Symposium, pp. 27-37, 2004.

[6] V. Jelicic, M. Magno, D. Brunelli, V. Bilas, and L. Benini, "Analytic comparison of wake-up receivers for wsns and benefits over the wake-on radio scheme," in Proceedings of the 7th ACM workshop on Performance monitoring and measurement of heterogeneous wireless and wired networks, pp. 99-106, ACM, 2012. 
[7] M. Magno and L. Benini, "An ultra low power high sensitivity wakeup radio receiver with addressing capability," in Wireless and Mobile Computing, Networking and Communications (WiMob), 2014 IEEE 10th International Conference on, pp. 92-99, IEEE, 2014.

[8] D. Spenza, M. Magno, S. Basagni, L. Benini, M. Paoli, and C. Petrioli, "Beyond Duty Cycling: Wake-up Radio with Selective Awakenings for Long-lived Wireless Sensing Systems," 2015.

[9] J. Ansari, D. Pankin, and P. Mahonen, "Radio-triggered Wake-ups with Addressing Capabilities for Extremely Low Power Sensor Network Applications," International Journal of Wireless Information Networks, vol. 16, pp. 118-130, Sept. 2009.

[10] J. Oller, I. Demirkol, J. Casademont, J. Paradells, G. Gamm, and L. Reindl, "Performance Evaluation and Comparative Analysis of SubCarrier Modulation Wake-up Radio Systems for Energy-Efficient Wireless Sensor Networks," Sensors, vol. 14, pp. 22-51, Dec. 2013.

[11] B. Van der Doorn, W. Kavelaars, and K. Langendoen, "A prototype low-cost wakeup radio for the $868 \mathrm{MHz}$ band," International Journal of Sensor Networks, vol. 5, no. 1, pp. 22-32, 2009.

[12] “TLV3691 Datasheet," tech. rep., Texas Instruments, 2013.

[13] "PIC24f16ka102 Family Data Sheet," tech. rep., Microchip, 2011. 